\title{
The FERRUM project: Experimental transition probabilities from highly excited even 5 s levels in $\mathrm{Cr}$ II
}

\author{
L. Engström ${ }^{1}$, H. Lundberg ${ }^{1}$, H. Nilsson ${ }^{2}$, H. Hartman ${ }^{2,3}$, and E. Bäckström ${ }^{4}$ \\ 1 Department of Physics, Lund University, Box 118, 22100 Lund, Sweden \\ e-mail: lars.engstrom@fysik.1th.se \\ 2 Lund Observatory, Lund University, Box 43, 22100 Lund, Sweden \\ 3 Applied Mathematics and Material Science, Malmö University, 20506 Malmö, Sweden \\ ${ }^{4}$ Department of Physics, Stockholm University, AlbaNova University Center, 10691 Stockholm, Sweden
}

Received 6 August 2014 / Accepted 1 September 2014

\section{ABSTRACT}

\begin{abstract}
We report lifetime measurements of the five levels in the $3 \mathrm{~d}^{4}\left(\mathrm{a}^{5} \mathrm{D}\right) 5 \mathrm{~s} \mathrm{e}^{6} \mathrm{D}$ term in $\mathrm{Cr}$ II at an energy around $83000 \mathrm{~cm}^{-1}$, and $\log (g f)$ values for 38 transitions from the investigated levels. The lifetimes are obtained using time-resolved, laser-induced fluorescence on ions from a laser-produced plasma. Since the levels have the same parity as the low-lying states directly populated in the plasma, we used a two-photon excitation scheme. This process is greatly facilitated by the presence of the $3 \mathrm{~d}^{4}\left(\mathrm{a}^{5} \mathrm{D}\right) 4 \mathrm{p} \mathrm{z} \mathrm{z}^{6} \mathrm{~F}$ levels at roughly half the energy difference. The $f$-values are obtained by combining the experimental lifetimes with branching fractions derived using relative intensities from a hollow cathode lamp recorded with a Fourier transform spectrometer.
\end{abstract}

Key words. atomic data - methods: laboratory: atomic - techniques: spectroscopic

\section{Introduction}

This paper reports the first measurements of lifetimes for highly excited levels of even parity in Cr II. The data is obtained using time-resolved, laser-induced fluorescence (TR-LIF) on ions created in a laser-produced plasma. The lowest configurations in this ion are the even parity $3 \mathrm{~d}^{5}$ and $3 \mathrm{~d}^{4} 4 \mathrm{~s}$, extending up to an energy of about $50000 \mathrm{~cm}^{-1}$ (Sansonetti et al. 2012). To reach the new levels in the $3 \mathrm{~d}^{4}\left(\mathrm{a}^{5} \mathrm{D}\right) 5 \mathrm{~s} \mathrm{e}^{6} \mathrm{D}$ term at an energy around $83000 \mathrm{~cm}^{-1}$, we have used two-photon excitation from the low-lying even $3 \mathrm{~d}^{4}\left(\mathrm{a}^{5} \mathrm{D}\right) 4 \mathrm{~s} \mathrm{a}^{6} \mathrm{D}$ levels. The probability for this absorption process is greatly enhanced by the presence of $3 d^{4}\left(a^{5} D\right) 4 p z^{6} F$ levels at roughly half the energy difference between $\mathrm{a}^{6} \mathrm{D}$ and $\mathrm{e}^{6} \mathrm{D}$.

All previous lifetime measurements in $\mathrm{Cr}$ II have focused on levels in the first excited odd configuration $3 \mathrm{~d}^{4} 4 \mathrm{p}$, beginning at an energy of $48000 \mathrm{~cm}^{-1}$. Measurements using the beam-foil technique have been reported by Pinnington et al. (1973) and Engman et al. (1975). The TR-LIF method has also been used in a number of previous studies (Schade et al. 1990; Bergeson \& Lawler 1993; Pinnington et al. 1993; Nilsson et al. 2006; and Gurell et al. 2010), where the $\mathrm{Cr}^{+}$ions were produced in a fast ion beam, in a hollow cathode, and in laser-produced plasmas. By combining the measured lifetimes with branching fractions $(B F \mathrm{~s})$, usually obtained from intensity calibrated Fourier transform spectra, transition probabilities have been derived. For example, the most recent studies by Nilsson et al. (2006) and Gurell et al. (2010) report 119 and 145 transition probabilities, respectively, for lines from the $3 \mathrm{~d}^{4} 4 \mathrm{p}$ configuration. See Gurell et al. (2010) for additional references to investigations of the $4 \mathrm{p}$ states.

Studies of lifetimes and $f$-values for the transition metals are important in astrophysics, and radiative data in Cr II are of particularly interest since these lines are observed in a broad range of stellar and nebular spectra (Andrievsky et al. 1994; and Shevchenko 1994). For example, several chemically peculiar stars show unexpectedly high abundances of chromium (Rice \& Wehlau 1994; and López-García et al. 2001). The FERRUM project is an ongoing international collaboration to address the need for data on singly ionized iron group elements (Johansson et al. 2002).

To determine accurate abundances for high metallicity stars, lines with high excitation potential are desirable to avoid saturation effects. This is especially important for stars where the use of different lines yield significantly different abundances. In addition, highly excited lines such as the ones reported in this paper, can be used as probes of deviations from local thermodynamic equilibrium (LTE) in stellar atmospheres. With known $f$-values, the highly excited lines benchmark the stellar atmospheres and aid in the determination of excitation distributions (Wood et al. 2014). A search was made for the lines in HD 84937, the solar type star $\alpha$ Cen A and the sun (Hinkle et al. 2005; Moore et al. 1982), but the temperature and the Boltzmann factor were too small to determine an abundance using these lines.

\section{Laboratory measurements}

\subsection{Lifetimes}

The TR-LIF setup at Lund High Power Laser Facility contains two Nd:YAG lasers operating at $10 \mathrm{~Hz}$. The frequency doubled output from one of them (Continuum Surelite) is focused onto a rotating chromium target placed inside a vacuum chamber (residual pressure about $10^{-5}$ mbar) to generate the ablation plasma. The second laser is an injection seeded and Q-switched Continuum NY-82. The $532 \mathrm{~nm}$ output was temporally compressed using stimulated Brillouin scattering in water before 


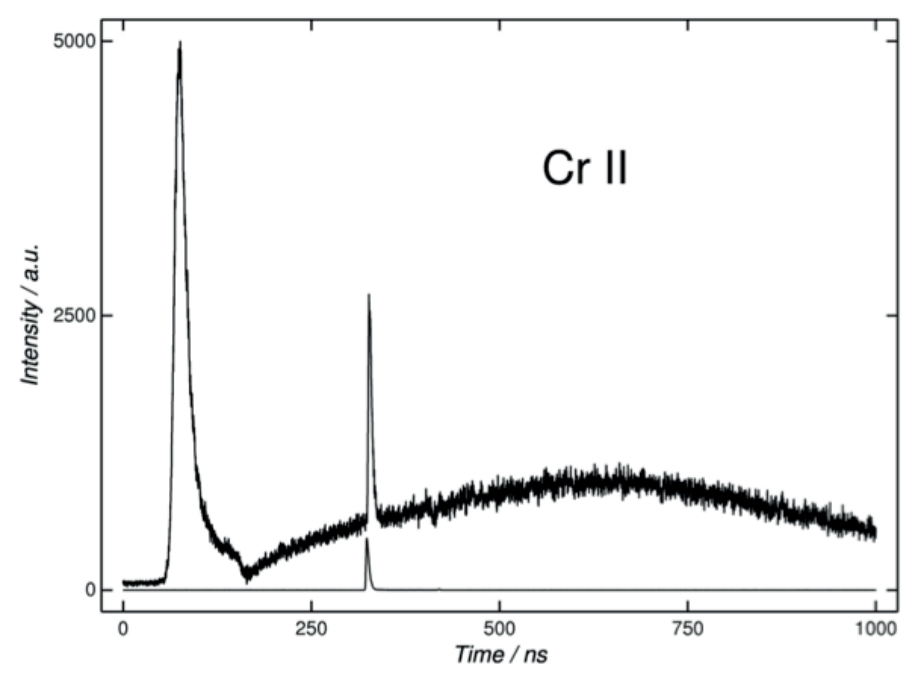

Fig. 1. TR-LIF measurement of the decay of the $5 \mathrm{~s}^{6} \mathrm{D}_{9 / 2}$ level in $\mathrm{Cr}$ II at $83241 \mathrm{~cm}^{-1}$ in a laser-produced plasma. The intense peak is the $532 \mathrm{~nm}$ Nd-YAG laser used to produce the plasma. The bottom peak is the delayed excitation laser pulse resulting in the observed fluorescence signal on top of the plasma background.

pumping a dye laser (Continuum Nd-60) using Rh 590, to optimize the laser intensity around $565 \mathrm{~nm}$. This output was then frequency doubled in a KDP crystal to obtain about $1 \mathrm{~mJ}$ of the $283 \mathrm{~nm}$ radiation needed for the two-photon excitation. Since two-photon absorption is a nonlinear process the probability is strongly dependent on the laser intensity. Thus, in addition to optimizing the laser power we carefully adjusted a quartz lens ( $f=150 \mathrm{~mm}$ ) placed just outside the vacuum chamber to obtain a sharp focus in the middle of the ablation plasma a few $\mathrm{mm}$ above the chromium target. Since different ionization stages produced in the plasma have different velocities, they can be separated by selecting an appropriate delay time between the ablation and the excitation pulses. The delay time turned out to be very critical in this experiment and virtually all measurements were made with a delay of $160 \mathrm{~ns}$. The laser-induced fluorescence was detected by a $1 / 8 \mathrm{~m}$ monochromator with a $280 \mu \mathrm{m}$ wide entrance slit oriented parallel to the excitation laser beam and perpendicular to the ablation laser. The fluorescence signal was recorded with a microchannel plate photomultiplier tube (Hamamatsu R3809U) with a rise time of 0.2 ns and digitized by a Tektronix oscilloscope with $2.5 \mathrm{GHz}$ analog bandwidth. A second channel on the oscilloscope sampled the excitation laser pulse shape measured by a fast photodiode. Figure 1 illustrates the timing of the lasers and the fluorescence signal.

Figure 2 gives a schematic overview of the energy terms and transitions involved in this study and Table 1 presents the experimental settings. The $3 \mathrm{~d}^{4} 5 \mathrm{~s} \mathrm{e}^{6} \mathrm{D}$ levels were excited by twophoton absorption from the low-lying, metastable $3 d^{4} 4 s$ a ${ }^{6} \mathrm{D}$ levels around $12000 \mathrm{~cm}^{-1}$. The energy difference is thus about $70700 \mathrm{~cm}^{-1}$, corresponding to two $282 \mathrm{~nm}$ photons, and the presence of the $3 d^{4} 4 p z^{6} F$ levels close to halfway in-between (within a few $100 \mathrm{~cm}^{-1}$ ) greatly enhanced the two-photon absorption probability. Only $\Delta J=0$ excitations could be observed. Since the fine structure separations in the $4 \mathrm{~s}$ and $5 \mathrm{~s} \mathrm{e}{ }^{6} \mathrm{D}$ terms are essentially determined by the common $3 d^{4}{ }^{5} \mathrm{D}$ parent, the excitation wavelengths are very close (see Table 1) and it was not possible to resolve the $e^{6} \mathrm{D}_{1 / 2}$ and $\mathrm{e}^{6} \mathrm{D}_{3 / 2}$ levels.

The most intense fluorescence channels from the $5 \mathrm{~s} \mathrm{e}^{6} \mathrm{D}$ levels are to $4 p z^{6} F$ followed by those to $4 p z^{6} P$ and $z^{6} D$. The transitions occur at 282, 289, and $299 \mathrm{~nm}$ (see Fig. 2 and

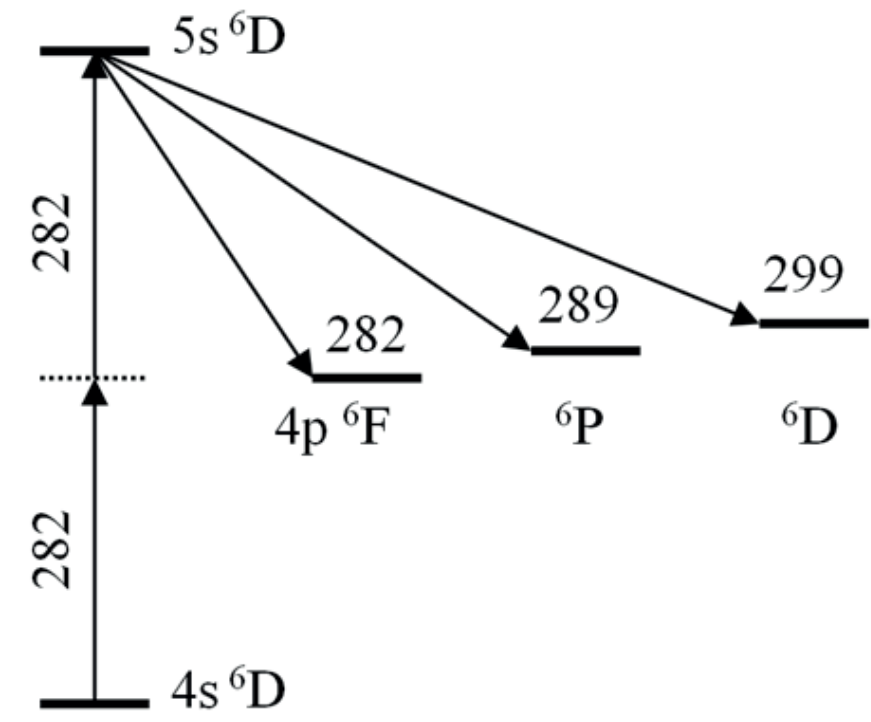

Fig. 2. Schematic term system of the $3 \mathrm{~d}^{4}\left(\mathrm{a}^{5} \mathrm{D}\right) \mathrm{n} \ell^{6} \mathrm{~L}$ terms in Cr II involved in this investigation. Wavelengths for the transitions are indicated in $\mathrm{nm}$.

Table 1), and were always measured in the second spectral order of the monochromator. In the case of the $e^{6} D_{9 / 2}$ level, we could observe the decay in all three channels, whereas for the other fine structure levels only the first two were intense enough to allow reliable measurements. In the $\mathrm{z}^{6} \mathrm{~F}-\mathrm{e}^{6} \mathrm{D}$ channel a minor problem occurred because of the proximity of the excitation and detection wavelengths. Thus, it was not possible to completely isolate the fluorescence from the very intense excitation laser light. To compensate for this, we also measured the scattered laser light with the ablation laser turned off and subtracted this signal from the observed decay curve before the lifetime analysis. This is illustrated for the $e^{6} \mathrm{D}_{7 / 2}$ level in Fig. 3. In this case, the excitation wavelength was $282.65 \mathrm{~nm}$ and the detection was at $281 \mathrm{~nm}$ with a instrumental line width of $0.5 \mathrm{~nm}$ in the second spectral order.

Each decay curve as well as the temporal shape of the excitation pulse was averaged over 1000 laser shots. The measured excitation pulse width (FWHM) was about $3 \mathrm{~ns}$, but since the $e^{6} \mathrm{D}$ levels were excited in a two-photon process the final analyses of the decay curves utilized the square of the measured pulse, which then had a temporal width of 2 ns. The lifetimes were extracted using a slightly modified version of the program DECFIT (Palmeri et al. 2008) by fitting the fluorescence data with a single exponential convoluted with the square of the measured laser pulse and a constant background. A typical example is shown in Fig. 3, where we have also included a measurement of the scattered laser light that was subtracted before the decay analysis. The final lifetimes given in Table 1 are the averages of between 16 and 22 measurements, performed at several different occasions. No systematic differences were found between the different detection channels. In addition, we note that the decay curves for the two unresolved levels, $e^{6} D_{1 / 2}$ and $e^{6} D_{3 / 2}$, showed no trace of a multiexponential behavior, indicating that the two lifetimes cannot be too different. The quoted uncertainties in the lifetimes include the statistical uncertainties as well as the variation of the results between the repeated measurements.

Table 1 also includes theoretical lifetimes obtained by Kurucz (1995) and by Raasen \& Uylings (1997). Both investigations are semiempirical in the sense that the radial integrals are treated as adjustable parameters that are optimized by fitting 
Table 1. Experimental details and the measured lifetimes of the $5 \mathrm{~s}{ }^{6} \mathrm{D}$ levels in Cr II.

\begin{tabular}{lllllcll}
\hline \hline $\begin{array}{l}\text { Upper level }^{a} \\
5 \mathrm{~s}^{6} \mathrm{D}_{J}\end{array}$ & $J$ & $\begin{array}{l}\text { Lower level }^{a} \\
4 \mathrm{~s}^{6} \mathrm{D}_{J}\end{array}$ & $J$ & Excitation $^{b}$ & Detection $^{c}$ & $\tau_{\text {exp }} / \mathrm{ns}$ & $\tau_{\text {th }} / \mathrm{ns}$ \\
$E / \mathrm{cm}^{-1}$ & $1 / 2$ & 11962 & $1 / 2$ & 282.680 & 279 & $2.5 \pm 0.2$ & $2.95^{d}, 2.83^{e}$ \\
\hline 82692 & $3 / 2^{f}$ & 12032 & $3 / 2$ & & & & \\
82763 & $5 / 2$ & 12148 & $5 / 2$ & 282.669 & 280 & $2.5 \pm 0.2$ & $2.94^{d}, 2.85^{e}$ \\
\hline 82881 & & & & & 290 & & \\
\hline 83041 & $7 / 2$ & 12303 & $7 / 2$ & 282.653 & 281 & $2.6 \pm 0.2$ & $2.94^{d}, 2.84^{e}$ \\
& & & & & 290 & & \\
\hline 83240 & $9 / 2$ & 12496 & $9 / 2$ & 282.629 & 282 & $2.6 \pm 0.2$ & $2.93^{d}, 2.86^{e}$ \\
& & & & & 289 & & \\
\hline
\end{tabular}

Notes. ${ }^{(a)}$ Sansonetti et al. (2012); ${ }^{(b)}$ Two-photon excitation using this wavelength; ${ }^{(c)}$ Transitions at 280 nm connect to $4 p z^{6} \mathrm{~F}$, those around $290 \mathrm{~nm}$ to $4 \mathrm{p} \mathrm{z}{ }^{6} \mathrm{P}$ and $299 \mathrm{~nm}$ to $4 \mathrm{p} \mathrm{z}^{6} \mathrm{D}$ (see Fig. 2). All measurements were made in the second spectral order; ${ }^{(d)}$ Semiempirical superpositionof-configurations calculation by Kurucz (1995); ${ }^{(e)}$ Semiempirical calculation using orthogonal operators by Raasen \& Uylings (1997); ${ }^{(f)}$ The $J=1 / 2$ and $3 / 2$ fine structure levels in $\mathrm{e}^{6} \mathrm{D}$ could neither be separated in the excitation nor in the detection channels.

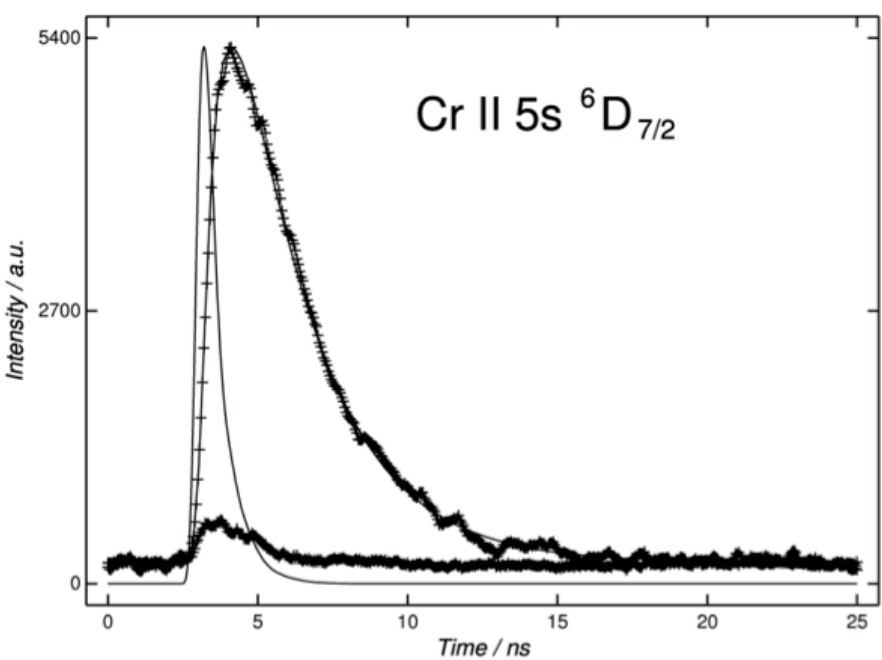

Fig. 3. Decay of the $5 \mathrm{~s}^{6} \mathrm{D}_{7 / 2}$ level in Cr II at $83041 \mathrm{~cm}^{-1}$ following twophoton excitation from the low-lying metastable $4 \mathrm{~s}^{6} \mathrm{D}_{7 / 2}$ level. The decay and the scattered excitation laser contribution at $281 \mathrm{~nm}$ are plotted with + signs. The square of the recorded laser pulse and the fitted decay curve are shown as solid lines.

to the experimental energies. This should improve the transition energies as well as the eigenvector representations. Kurucz used a modified version of the Cowan codes (Cowan 1981), whereas Raasen \& Uylings (1997) applied the orthogonal operator formalism. There is fairly good agreement between both calculations and the experimental data (13-18\%), although the results by Raasen \& Uylings (1997) are consistently closer to our measurements. Furthermore, neither the experimental nor the theoretical lifetimes indicate any significant $J$-dependence of the values. We also note that while the experimental lifetimes in the $3 \mathrm{~d}^{4} 4 \mathrm{p}$ configuration are consistently longer than those calculated by Kurucz (1995) by, on the average, 37\% (Nilsson et al. 2006) and $22 \%$ (Gurell et al. 2010) in the present case the situation is reversed and the experimental values are consistently shorter, by about $15 \%$. A similar comparison between the $3 d^{4} 4 p$ lifetimes in Gurell et al. (2010) and the theoretical results by Raasen \& Uylings (1997) shows a perfect agreement within the error bars in all cases.

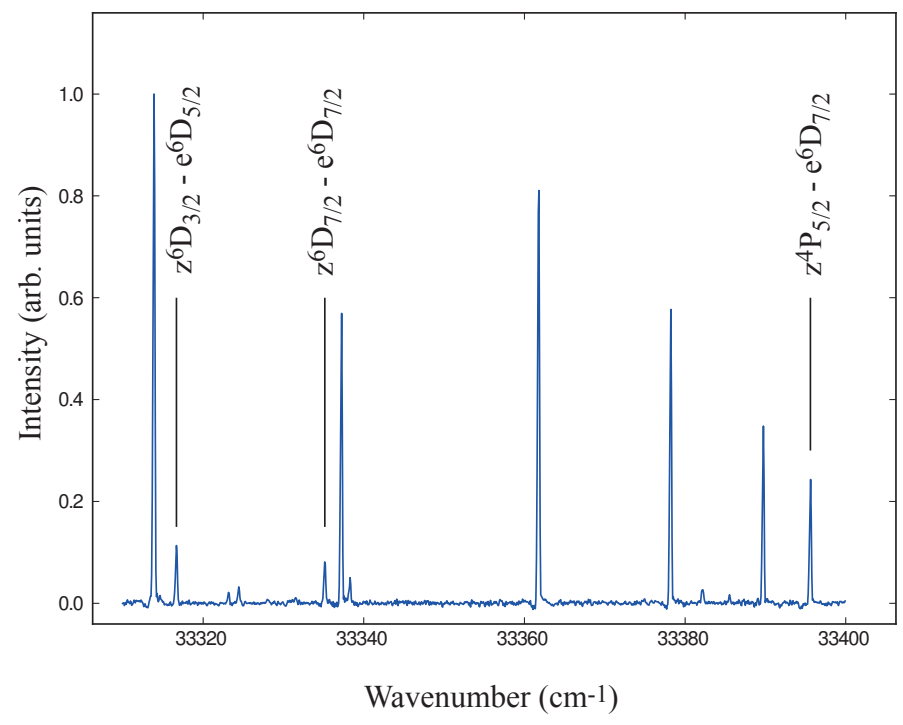

Fig. 4. Part of a recorded FTS-spectrum between $33300-33400 \mathrm{~cm}^{-1}$, including the Cr II lines $\mathrm{z}^{6} \mathrm{D}_{3 / 2}-\mathrm{e}^{6} \mathrm{D}_{5 / 2}, \mathrm{z}^{6} \mathrm{D}_{7 / 2}-\mathrm{e}^{6} \mathrm{D}_{7 / 2}$, and $\mathrm{z}^{4} \mathrm{P}_{5 / 2}-$ $\mathrm{e}^{6} \mathrm{D}_{7 / 2}$ at $\lambda \lambda 3000.63,2993.54$, and $2998.97 \AA$ respectively.

\subsection{Branching fractions (BFs)}

The $B F$ s were measured from spectra recorded with the Chelsea Instrument FT500 UV FT spectrometer at Lund Observatory. The light source was a Hollow Cathode (HC) discharge lamp. The HC was loaded with a pure chromium cathode and operated with neon as carrier gas at pressures between 0.5 and 2 Torr. The applied currents varied in the range $0.1-0.7 \mathrm{~A}$. The best signalto-noise $(\mathrm{S} / \mathrm{N})$ for the $4 \mathrm{p}-5 \mathrm{~s}$ transitions was obtained at $0.7 \mathrm{~A}$ and 2 Torr.

The HC lamp provides an intensity stable emission spectrum over several hours, which enabled us to perform up to 20 scans, that were co-added to give a higher $\mathrm{S} / \mathrm{N}$ ratio. Figure 4 shows a small part of the observed spectrum, including three $\mathrm{Cr}$ II lines from the investigated upper levels. All spectra were recorded between 20000 and $40000 \mathrm{~cm}^{-1}$, however, since the noise level in the spectrum depends on the total intensity, we limited the wavenumber coverage as much as possible. By using a standard colored glass filter (UG5) and the sensitivity of the detector, a Hamamatsu PMT 1P28, the wavelength region 
Table 2. Experimental and theoretical branching fractions in Cr II.

\begin{tabular}{|c|c|c|c|c|c|c|c|}
\hline $\begin{array}{l}\text { Upper } \\
\text { level }^{a}\end{array}$ & $\tau / \mathrm{ns}$ & $\begin{array}{l}\text { Lower } \\
\text { level }^{a}\end{array}$ & $\lambda_{\mathrm{air}^{a}}{ }^{a} / \AA$ & $B F_{\mathrm{th}}{ }^{b}$ & $B F_{\text {exp }}$ & $\log (g f)$ & Unc \% \\
\hline \multirow[t]{7}{*}{${ }^{6} \mathrm{D}_{1 / 2}$} & \multirow[t]{8}{*}{$2.5(2)$} & $\mathrm{z}^{6} \mathrm{~F}_{1 / 2}$ & 2787.12347 & .279 & $.254(13)$ & -.627 & 15 \\
\hline & & $z^{6} F_{3 / 2}$ & 2793.49677 & .218 & $.221(15)$ & -.684 & 17 \\
\hline & & $\mathrm{z}^{6} \mathrm{P}_{3 / 2}$ & 2915.18232 & .176 & $.207(9)$ & -.675 & 12 \\
\hline & & $\mathrm{z}^{4} \mathrm{P}_{1 / 2}$ & 2945.2753 & .0215 & $.0496(19)$ & -1.29 & 21 \\
\hline & & $\mathrm{z}^{4} \mathrm{P}_{3 / 2}$ & 2967.728 & .132 & $.0882(11)$ & -1.03 & 13 \\
\hline & & $\mathrm{z}^{6} \mathrm{D}_{1 / 2}$ & 3011.2368 & .0451 & $.0355(16)$ & -1.41 & 18 \\
\hline & & $\mathrm{z}^{6} \mathrm{D}_{3 / 2}$ & 3017.78 & .129 & $.145(8)$ & -.802 & 12 \\
\hline Residual & & & & $3.4 \times 10^{-4}$ & & & \\
\hline \multirow[t]{9}{*}{${ }^{6} \mathrm{D}_{3 / 2}$} & \multirow[t]{9}{*}{$2.5(2)$} & $\mathrm{z}^{6} \mathrm{~F}_{3 / 2}$ & 2787.91434 & .206 & $.270(8)$ & -.299 & 11 \\
\hline & & $z^{6} F_{5 / 2}$ & 2798.46148 & .167 & $.149(8)$ & -.553 & 11 \\
\hline & & $\mathrm{z}^{6} \mathrm{P}_{3 / 2}$ & 2909.10501 & .141 & $.168(7)$ & -.466 & 11 \\
\hline & & $\mathrm{z}^{6} \mathrm{P}_{5 / 2}$ & 2916.9279 & .0338 & $.0539(14)$ & -.958 & 16 \\
\hline & & $\mathrm{z}^{4} \mathrm{P}_{1 / 2}$ & 2939.0733 & .0757 & $.0287(17)$ & -1.23 & 19 \\
\hline & & $\mathrm{z}^{6} \mathrm{D}_{5 / 2}$ & 2992.07043 & .0920 & $.137(6)$ & -.533 & 10 \\
\hline & & $z^{6} D_{1 / 2}$ & 3004.7478 & .157 & $.0754(6)$ & -.787 & 10 \\
\hline & & $\mathrm{z}^{4} \mathrm{P}_{5 / 2}$ & 3024.1603 & .0364 & $.0275(8)$ & -1.22 & 11 \\
\hline & & & & .091 & & & \\
\hline \multirow[t]{9}{*}{${ }^{6} \mathrm{D}_{5 / 2}$} & \multirow[t]{10}{*}{$2.5(2)$} & $\mathrm{z}^{6} \mathrm{~F}_{5 / 2}$ & 2789.29198 & .169 & $.129(9)$ & -.441 & 12 \\
\hline & & $\mathrm{z}^{6} \mathrm{~F}_{7 / 2}$ & 2803.91770 & .221 & $.245(5)$ & -.159 & 10 \\
\hline & & $\mathrm{z}^{6} \mathrm{P}_{3 / 2}$ & 2899.1907 & .115 & $.171(6)$ & -.286 & 10 \\
\hline & & $z^{6} P_{5 / 2}$ & 2906.96698 & .117 & $.123(6)$ & -.429 & 10 \\
\hline & & $\mathrm{z}^{4} \mathrm{P}_{3 / 2}$ & 2951.1448 & .0515 & $.0255(15)$ & -1.10 & 17 \\
\hline & & $\mathrm{z}^{6} \mathrm{D}_{5 / 2}$ & 2981.5903 & .0464 & $.0563(8)$ & -.744 & 11 \\
\hline & & $z^{6} D_{3 / 2}$ & 3000.63027 & .112 & $.0974(6)$ & -.501 & 10 \\
\hline & & $z^{6} D_{7 / 2}$ & 3007.97320 & .101 & $.0861(6)$ & -.552 & 10 \\
\hline & & $\mathrm{z}^{4} \mathrm{P}_{5 / 2}$ & 3013.471 & .0119 & $.00961(17)$ & -1.50 & 19 \\
\hline Residual & & & & .056 & & & \\
\hline \multirow[t]{8}{*}{${ }^{6} \mathrm{D}_{7 / 2}$} & \multirow[t]{9}{*}{$2.6(2)$} & $\mathrm{z}^{6} \mathrm{~F}_{7 / 2}$ & 2791.37318 & .125 & $.146(9)$ & -.279 & 12 \\
\hline & & $z^{6} F_{9 / 2}$ & 2809.99583 & .281 & $.267(5)$ & -.0122 & 9 \\
\hline & & $\mathrm{z}^{6} \mathrm{P}_{5 / 2}$ & 2893.48608 & .197 & $.161(5)$ & -.208 & 9 \\
\hline & & $\mathrm{z}^{6} \mathrm{P}_{7 / 2}$ & 2905.34374 & .0679 & $.0825(7)$ & -.493 & 11 \\
\hline & & $\mathrm{z}^{6} \mathrm{D}_{5 / 2}$ & 2967.40937 & .0622 & $.0662(8)$ & -.571 & 11 \\
\hline & & $z^{6} D_{7 / 2}$ & 2993.54120 & .133 & $.130(5)$ & -.271 & 9 \\
\hline & & $\mathrm{z}^{4} \mathrm{P}_{5 / 2}$ & 2998.97015 & .0513 & $.0447(7)$ & -.732 & 10 \\
\hline & & $z^{6} D_{9 / 2}$ & 3010.90430 & .0586 & $.0791(6)$ & -.481 & 10 \\
\hline Residual & & & & .024 & & & \\
\hline \multirow[t]{6}{*}{${ }^{6} \mathrm{D}_{9 / 2}$} & \multirow[t]{7}{*}{$2.6(2)$} & $\mathrm{z}^{6} \mathrm{~F}_{9 / 2}$ & 2794.35678 & .0706 & $.0999(11)$ & -.347 & 13 \\
\hline & & $\mathrm{z}^{6} \mathrm{~F}_{11 / 2}$ & 2816.95939 & .349 & $.289(4)$ & .121 & 9 \\
\hline & & $\mathrm{z}^{6} \mathrm{P}_{7 / 2}$ & 2888.62821 & .275 & $.241(5)$ & .0640 & 9 \\
\hline & & $z^{6} D_{7 / 2}$ & 2975.8007 & .0607 & $.0558(7)$ & -.546 & 10 \\
\hline & & $z^{6} D_{9 / 2}$ & 2992.95629 & .234 & $.298(4)$ & .187 & 9 \\
\hline & & $\mathrm{z}^{4} \mathrm{~F}_{9 / 2}$ & 3194.194 & .00342 & .00893(19) & -1.28 & 21 \\
\hline Residual & & & & .0078 & & & \\
\hline
\end{tabular}

References. ${ }^{(a)}$ Sansonetti et al. (2012); ${ }^{(b)}$ Raasen \& Uylings (1997).

was thus restricted to $26000-36000 \mathrm{~cm}^{-1}(277-375 \mathrm{~nm})$. The spectra were intensity calibrated using a Deuterium lamp with known relative spectral radiance measured at the PhysicalischTechnische Bundesanstalt, Berlin, Germany. The spectral lines were fitted with Gaussian profiles to determine the integrated intensity and its uncertainty using the free and available software GFit (Engström 1998).

The measured intensities were converted to $B F$ s through:

$B F_{i k}=I_{i k} / \Sigma_{n} I_{i n}$,

where $I_{i k}$ is the calibrated intensity of a transition from the upper level $i$ to a lower level $k$, and the sum is the total intensity for all lines coming from level $i$. Lines too weak to be measured in the spectra were included in the sum using the theoretical branching fractions from Raasen \& Uylings (1997). Table 2 presents experimental and theoretical $B F$ s together with $\log (g f)$ values obtained by combining the measured $B F$ s with the experimental lifetimes in Table 1. The quantity denoted "residual" in Table 2 is the sum of the $B F$ s taken from the theoretical calculations by Raasen \& Uylings (1997) for lines too weak to be observed. In general, the weak missing branches fall in the visible part of the spectrum, with a maximum residual of $9.1 \%$ for the ${ }^{6} \mathrm{D}_{3 / 2}$ level. The uncertainty in the $B F$ s includes contributions from the intensity measurements and calibration, and a $50 \%$ estimated uncertainty in the residual. The uncertainty in the $\log (g f)$-values is derived by adding the uncertainty of the measured lifetimes. Further discussion concerning error analyses can be found in Sikström et al. (2002). 


\section{Summary}

In this paper, we report an experimental study of the decay of the most highly excited levels in Cr II investigated so far. These levels, at about $60 \%$ of the ionization energy, should be possible to use as probes of deviations from local thermodynamic equilibrium (LTE) in stellar atmospheres and aid in the determination of excitation distributions. The data consists of the lifetimes of the five levels in the $3 \mathrm{~d}^{4}\left(\mathrm{a}^{5} \mathrm{D}\right) 5 \mathrm{~s} \mathrm{e}^{6} \mathrm{D}$ term and $\log (g f)$ values for 38 transitions from the investigated levels. The results are obtained by combining lifetime measurements, using the TR-LIF technique with two photon excitation, and branching fractions derived using relative intensities from a hollow cathode lamp recorded with a Fourier transform spectrometer.

Acknowledgements. We acknowledge Chris Sneden and Matthew Alvarez, (University of Texas) and their effort to model the present lines in the metalpoor star HD 84937. This work was supported by the Swedish Research Council through the Linnaeus grant to the Lund Laser Centre and the Knut and Alice Wallenberg Foundation. H.H. gratefully acknowledges the grant no 621-20114206 from the Swedish Research Council.

\section{References}

Andrievsky, S. M., Kovtyukh, V. V., \& Usenko, A. 1994, A\&A, 281, 465 Bergeson, S. D., \& Lawler, J. E. 1993, ApJ, 408, 382

Cowan, R. D. 1981, The Theory of Atomic Structure and Spectra (Berkeley Univ. of California Press)
Engman, B., Gaupp, A., Curtis, L. J., \& Martinsson, I. 1975, Phys. Scripta, 12, 220

Engström, L. 1998, Lund Reports in Atomic Physics (LRAP-232), Atomic Physics, Lund University, http://kurslab-atom. fysik.1th.se/Lars/ GFit/Html/index.html

Gurell, J., Nilsson, H., Engström, L., et al. 2010, A\&A, 511, A68

Hinkle, K., Wallace, L., Valenti, J., \& Ayres, T. 2005, Ultraviolet Atlas of the Arcturus Spectrum, 1150-3800 A (San Francisco: ASP)

Kurucz, R. L. 1995, Atomic spectral line data from CD-ROM No. 23 http: //wWw. cfa.harvard. edu/amp/ampdata/kurucz23/sekur.html Johansson, S., Derkatch, A., Donnelly, M., et al. 2002, Phys. Scripta, T100, 71

López-García, Z., Adelman, S. J., \& Pintado, O. I. 2001, A\&A, 367, 859

Moore, C. E., Tousey, R., \& Brown, C. M. 2005, The solar spectrum 3069-2095 angstroms from the echelle spectrograph flown in 1961 and 1964 (Interim Report Naval Research Lab., Washington, DC. Center for Space Research)

Nilsson, H., Ljung, G., Lundberg, H., \& Nielsen, K. E. 2006, A\&A, 445, 1165

Palmeri, P., Quinet, P., Fivet, V., et al. 2008, Phys. Scripta, 78, 015304

Pinnington, E. H., Lutz, H. O., \& Carriaveao, G. W. 1973, Nucl. instrum. Methods, 110, 55

Pinnington, E. H., Ji, Q., Guo, B., et al. 1993, Can. J. Phys., 71, 470

Raasen, A. J. J., \& Uylings, P. H. M. 1997, unpublished data available at: ftp: //ftp.wins. uva.nl/pub/orth/chromium

Rice, J. B., \& Wehlau, W. H. 1994, A\&A, 291, 825

Sansonetti, C. J., Nave, G., Reader, J., \& Kerber, F. 2012, ApJSS, 202, 15

Schade, W., Mundt, B., \& Helbig, V. 1990, Phys. Rev. A, 42, 3

Shevchenko, V. S. 1994, Astron. Zh., 71, 572

Sikström, C. M., Nilsson, H., Litzén, U., Blom, A., \& Lundberg, H. 2002, J. Quant. Spectr. Rad. Transf., 74, 355

Wood, M. P., Lawler, J. E., Sneden, C., \& Cowan, J. J. 2014, ApJ, 211, 20 